\title{
Correction to "The Electronic Primary Care Research Network (ePCRN): A New Era in Practice-based Research"
}

In the abovementioned article (Peterson K, Fontaine P, Speedie S. J Am Board Fam Med 2006;19:93-7), the following material was inadvertently omitted:

Funding: This project was funded in whole or in part with federal funds from the National Institutes of Health, under contract no. HHS268N200425212C, "Re-engineering the Clinical Research Enterprise."

Acknowledgements: The ePCRN would like to acknowledge the following core networks, network directors, coordinators, and members: AAFP National Research Network (NRN), Leawood, KS; Alabama Practice Based Research Network (APBRN), Birmingham, AL; Indiana Family Practice Research Network (INET), Indianapolis, IN; Minnesota Academy of Family Physicians Research Network (MAFPRN), Minneapolis, MN; Oklahoma Physician Resource Research Network (OKPRN), Oklahoma City, OK; Penn State Ambulatory Research Network (PSARN), Hershey, PA; State Network of Colorado Ambulatory Practices \& Partners (SNOCAP), Denver, CO; South Texas Ambulatory Research Network (STARNET), San Antonio, TX; South Florida Primary Care Practice Based Research Network (SoFlaPBRN), Miami, FL; Upstate New York Practice Based Research Network (UNY-NET), Buffalo, NY.

We apologize for any confusion or inconvenience caused by this omission. 